\title{
Quantifying the extent of recrossing flux for quantum systems
}

\author{
Michael S. Small, Cristian Predescu, ${ }^{*}$ and William H. Miller \\ Department of Chemistry and Kenneth S. Pitzer Center for \\ Theoretical Chemistry, University of California, Berkeley, CA 94720
}

(Dated: April 14, 2005)

\begin{abstract}
We present arguments demonstrating that the Miller, Schwartz, and Tromp (MST) correlation function is the only computationally reasonable choice with regard to minimizing the extent of recrossing flux. However, using accurate numerical results, we point out that the MST flux-flux correlation function almost always exhibits non-vanishing negative parts, even for the simplest physical systems. We argue that, in order to best handle the residual recrossing flux, one must not rely on the "no recrossing" assumption in the development of quantum transition state theories. To provide accurate numerical examples, we derive the analytical expressions for the the flux-flux correlation and spectral functions for the symmetric Eckart and rectangular potential barriers.
\end{abstract}

Keywords: Eckart barrier, rectangular barrier, quantum transition state theory, recrossing

*Electronic address: cpredescu@comcast. net 


\section{INTRODUCTION}

Within the realm of classical mechanics, transition state theory (TST) is a well-defined variational theory, the computational usefulness of which has been established in numerous studies. Wigner's variational principle $[1,2]$ holds that the instantaneous flux through any surface separating the reactants from the products is an upper bound to the exact reaction rate. Indeed, of the trajectories starting out on the dividing surface, only a fraction will stay on the product side in the infinite future [3, 4]. After crossing the dividing surface once or several times, the remaining trajectories end up on the reactant side and are, therefore, non-reactive. Classical transition state theory is exact for those surfaces and systems for which there is no recrossing flux. For example, as argued by Pechukas and McLafferty [4], recrossing flux vanishes for sufficiently low temperatures. For one-dimensional systems, for which the dynamics is completely determined by the conservation of energy, placing the dividing surface at the top of the barrier eliminates all recrossing flux. Consequently, TST produces very accurate estimates for all multi-dimensional systems for which the separability assumption is a good approximation.

Unfortunately, despite the ongoing research effort on constructing quantum transition state theories for the last few decades, nothing has emerged that one can properly call a rigorous quantum TST [5, 6]. Variational formulations have been developed [7], but their usefulness is extremely limited. A variational principle is computationally useful only if it provides exact answers for a special yet sufficiently general class of systems. For classical systems, we have presented such examples in the preceding paragraph. For quantum systems, examples are virtually non-existent. For instance, the assumption of separability of the Hamiltonian about the saddle point, which is the hallmark of most more approximate classical TST's, is generally not a good approximation in the quantum world, even in the low temperature limit. The failure of the assumption of separability has led one of us to the development of what can be called the "direct" and "correct" theory for calculating rate constants [8], a theory that is equivalent to the quantum scattering theory. The resulting formula has been subsequently recast in terms of flux-flux correlation functions [9], thus enabling the development of more efficient numerical algorithms as well as establishing a connection with previous work of Yamamoto [10].

The hypothesis of no recrossing flux is formally equivalent to the existence of correlation 
functions that have no negative part. In the present paper, we demonstrate that quantum flux-flux correlation functions fail to exhibit vanishing negative parts even for the simplest physical systems: the one-dimensional symmetric barriers. We work with symmetric barriers because there is no ambiguity about the optimal location of the dividing surface: the optimal location is at the top of the barrier, which follows from the symmetry of the barrier and the fact that the potential decreases monotonically to the left and the right of the top of the barrier. Because of these special properties, a symmetric potential barrier represents the basic prototype for testing the "no recrossing" assumption.

In Section II, we demonstrate that the Miller, Schwartz, and Tromp (MST) flux-flux correlation function represents the optimal choice with respect to minimizing the magnitude of the primary recrossing frequencies. In Section III, for a rectangular barrier, we prove that there can be no correlation functions with vanishing negative parts. We utilize the result to argue that the MST flux-flux correlation function is the only reasonable candidate for the development of efficient numerical algorithms. In Section IV, we quantify the amount of recrossing for the symmetric Eckart barrier, which is defined by a smooth function and represents a more realistic example of a barrier. With the help of accurate numerical results, we demonstrate that the symmetric Eckart barrier is not free from recrossing, although the extent is small. We then argue that any efficient computational strategy must not attempt to improve on the MST flux-flux correlation function to further reduce the amount of recrossing flux. Rather, it must allow for the possibility that the correlation functions might unavoidable have non-vanishing negative parts. The presence of recrossing flux for the simplest, over-the-barrier dynamics constitutes a direct proof that, as opposed to the world of classical mechanics, there can be no exact quantum transition state theories. This conclusion is in agreement with the general experience on constructing quantum TST's, as summarized by Miller [5] and Garrett [6].

The accurate numerical results utilized in the present study are obtained from the analytical expressions for the flux-flux correlation and spectral functions for the symmetric rectangular and Eckart barriers. The derivation of the analytical formulas represents an integral part of the present paper. These expressions are important for testing the numerical stability of path-integral Monte Carlo algorithms that attempt to compute derivatives of the flux-flux correlation function at the origin [11]. Even for moderately large orders, such derivatives are difficult to obtain by standard discretization methods. However, because 
these derivatives represent the moments of the flux-flux spectral function, they can be accurately computed by numerical quadrature whenever analytical expressions for the spectral functions are available.

\section{QUANTIFYING THE EXTENT OF RECROSSING}

The most general correlation function that may be used for the computation of the quantum reaction rate has the form

$$
C_{F, \nu}(t)=\int_{0}^{\beta} \operatorname{tr}\left[e^{-(\lambda-i t / \hbar) H} \hat{F} e^{-(\beta-\lambda+i t / \hbar) H} \hat{F}\right] d \nu(\lambda) .
$$

In Eq. (1), $\hat{F}$ stands for the flux operator, $\beta=1 /\left(k_{B} T\right)$ is the inverse temperature, and $H$ denotes the Hamiltonian. The matrix element of the flux operator for a dividing surface located at the origin is given by

$$
\langle\psi|\hat{F}| \phi\rangle=\frac{\hbar}{2 m_{0} i}\left[\psi^{*}(0) \frac{d \phi}{d x}(0)-\frac{d \psi^{*}}{d x}(0) \phi(0)\right] .
$$

The quantity $d \nu(\lambda)$ is a measure on the interval $[0, \beta]$ that is positive and integrates to 1 . For example, for the Yamamoto correlation function [10], we have $d \nu(\lambda)=(1 / \beta) d \lambda$, whereas for the MST correlation function, we have $d \nu(\lambda)=\delta(\lambda-\beta / 2) d \lambda$, i.e., a Dirac delta function about $\beta / 2$.

The flux-flux correlation and spectral functions can be calculated at once by numerical quadrature provided that the matrix elements $|\langle E|\hat{F}| E\rangle|^{2}$ are known. Indeed, with the help of the spectral decomposition (we assume that the potential function is positive)

$$
e^{-\beta H}=\int_{0}^{\infty} e^{-\beta E}|E\rangle\langle E| d E
$$

the flux-flux correlation function is given by

$$
\begin{aligned}
C_{F, \nu}(t)=\int_{0}^{\beta} d \nu(\lambda) & \int_{0}^{\infty} d E \int_{0}^{\infty} d E^{\prime} e^{-(\lambda-i t / \hbar) E} \\
& \times e^{-(\beta-\lambda+i t / \hbar) E^{\prime}}\left|\left\langle E|\hat{F}| E^{\prime}\right\rangle\right|^{2} .
\end{aligned}
$$

The associated flux-flux spectral function, which is defined as its Fourier transform

$$
\bar{C}_{F, \nu}(\omega)=\frac{1}{2 \pi} \int_{\mathbb{R}} C_{F, \nu}(t) e^{-i \omega t} d t
$$


can be expressed in terms of the MST flux-flux spectral function as follows:

$$
\bar{C}_{F, \nu}(\omega)=\left[\int_{0}^{\beta} e^{-(\lambda-\beta / 2) \hbar \omega} d \nu(\lambda)\right] \bar{C}_{F}^{\mathrm{MST}}(\omega) .
$$

For $\omega \geq 0$, the MST flux-flux spectral function is given by [11]

$$
\bar{C}_{F}^{\mathrm{MST}}(\omega)=2 \hbar e^{-\beta \hbar \omega / 2} \int_{0}^{\infty} d E e^{-\beta E}|\langle E+\omega \hbar|\hat{F}| E\rangle|^{2}
$$

and extended to negative frequencies by symmetry.

The quantum reaction rate, which is defined as

$$
\begin{array}{r}
k(T) Q_{r}(T)=\int_{0}^{\infty} \operatorname{Re}\left[C_{F, \nu}(t)\right] d t \\
=\frac{1}{2} \int_{\mathbb{R}} C_{F, \nu}(t) d t=\pi \bar{C}_{F, \nu}(0),
\end{array}
$$

is then given by

$$
k(T) Q_{r}(T)=2 \pi \hbar \int_{0}^{\infty} d E e^{-\beta E}|\langle E|\hat{F}| E\rangle|^{2},
$$

and is seen to be independent of the particular choice of correlation function, as first demonstrated in Ref. 9. Because the quantum reaction rate is defined as the time integral of the real part of the correlation function, we make the standard convention that $C_{F, \nu}(t)$ refers to the real part of the actual correlation function.

In a recent paper [13], two of the present authors have related the quantity

$$
C_{F, \nu}(0)=\int_{\mathbb{R}} \bar{C}_{F, \nu}(\omega) d \omega
$$

to the extent to which the correlation functions oscillate. The argument utilized is that the oscillatory nature of the correlation function is due to the main primary frequencies, more precisely, to the magnitude of the peaks of the spectral function $\bar{C}_{F, \nu}(\omega)$ away from the origin. Mathematically, the argument can be understood as follows. An adequate measure of the extent of recrossing is given by the ratio

$$
p(\nu)=\int_{0}^{\infty}\left|C_{F, \nu}(t)\right| d t / \int_{0}^{\infty} C_{F, \nu}(t) d t,
$$

which takes the value 1 only if the correlation function is positive. The standard inequality

$$
\begin{array}{r}
\int_{0}^{\infty}\left|C_{F, \nu}(t)\right| d t=\frac{1}{2} \int_{-\infty}^{\infty}\left|C_{F, \nu}(t) e^{-i \omega t}\right| d t \\
\geq \frac{1}{2}\left|\int_{-\infty}^{\infty} C_{F, \nu}(t) e^{-i \omega t} d t\right|=\pi \bar{C}_{F, \nu}(\omega)
\end{array}
$$


implies

$$
p(\nu) \geq \sup _{\omega \in \mathbb{R}} \bar{C}_{F, \nu}(\omega) / \bar{C}_{F, \nu}(0) .
$$

In words, maxima of the spectral functions of intensities greater than the value at the origin automatically lead to recrossing. The non-zero frequencies for which such maxima are realized are called the main primary frequencies. As shown by the preceding equation, they constitute the main sources of recrossing. As a consequence, it is desirable that the correlation function and the dividing surface be chosen such that

$$
\sup _{\omega \in \mathbb{R}} \bar{C}_{F, \nu}(\omega)=\bar{C}_{F, \nu}(0)
$$

provided that such a choice exists.

We now establish the optimality of the MST correlation function in the sense of minimizing $\sup _{\omega \in \mathbb{R}} \bar{C}_{F, \nu}(\omega)$. The Cauchy-Schwartz inequality

$$
\begin{aligned}
& 1=\left[\int_{0}^{\beta} e^{-(\lambda-\beta / 2) \hbar \omega / 2} e^{+(\lambda-\beta / 2) \hbar \omega / 2} d \nu(\lambda)\right]^{2} \\
& \leq\left[\int_{0}^{\beta} e^{-(\lambda-\beta / 2) \hbar \omega} d \nu(\lambda)\right]\left[\int_{0}^{\beta} e^{+(\lambda-\beta / 2) \hbar \omega} d \nu(\lambda)\right]
\end{aligned}
$$

shows that either

$$
\int_{0}^{\beta} e^{-(\lambda-\beta / 2) \hbar \omega} d \nu(\lambda) \geq 1
$$

or

$$
\int_{0}^{\beta} e^{+(\lambda-\beta / 2) \hbar \omega} d \nu(\lambda) \geq 1 .
$$

Then, the symmetry of the MST spectral function and Eq. (3) imply that

$$
\sup _{\omega \in \mathbb{R}} \bar{C}_{F, \nu}(\omega) \geq \sup _{\omega \in \mathbb{R}} \bar{C}_{F}^{\mathrm{MST}}(\omega)
$$

and our optimality claim is proved. Eq. (10) demonstrates that, for the computationally unfavorable case for which Eq. (9) is not satisfied, one cannot remove the recrossing by optimizing the spectral function. In fact, at least in the sense of Eq. (10), any correlation function other than the MST one will actually exacerbate the amount of recrossing.

If Eq. (9) is satisfied by some correlation function, then it is automatically satisfied by the MST correlation function. In such a case, the optimal correlation function is in general not unique. Whether or not we can further reduce the amount of recrossing by directly minimizing the ratio $p(\nu)$ is an ill-posed question because the evaluation of $p(\nu)$ requires 
full knowledge of the correlation function. Notice that the utilization of the $\sup _{\omega \in \mathbb{R}} \bar{C}_{F, \nu}(\omega)$ criterion itself becomes a well-posed question only after we prove that we do not have to actually compute the quantity $\sup _{\omega \in \mathbb{R}} \bar{C}_{F, \nu}(\omega)$, in order to determine the optimal correlation function. Unfortunately, this is not the case if the ratio $p(\nu)$ is utilized as a criterion. Nevertheless, we expect that the amount of residual recrossing is minimal for all correlation functions that satisfy Eq. (9). We therefore conclude that, for all systems, the MST correlation function is a nearly optimal correlation function in the sense of minimizing $p(\nu)$ and the only optimal and universal choice in the sense of minimizing $\sup _{\omega \in \mathbb{R}} \bar{C}_{F, \nu}(\omega)$. Any correlation function that might exhibit less recrossing necessarily depends on the specifics of the particular system and has, therefore, limited computational value. We shall comment more about this observation in Section IV.

The $\sup _{\omega \in \mathbb{R}} \bar{C}_{F, \nu}(\omega)$ criterion cannot be utilized for the problem of determining the optimal dividing surface because, as mentioned before, it requires detailed knowledge of the structure of the spectral function, knowledge that is difficult to obtain from short-time information, only. Instead, the quantity

$$
C_{F, \nu}(0)=\int_{\mathbb{R}} \bar{C}_{F, \nu}(\omega) d \omega
$$

can be utilized to achieve similar results. The difference between the two criteria is precisely the difference between the approximation of functions in the uniform and the $L^{1}$ norms, on the real line. In more physical terms, we try to diminish the intensity rather than the magnitude of the main primary frequencies. Additional mathematical arguments related to the sensitivity of the short-time information with respect to the value of the quantum reaction rate have led the authors of Ref. 13 to propose the minimization of $C_{F, \nu}(0)$ against $\nu$ and $\hat{F}$ as a viable criterion for choosing a computationally optimal correlation function (by minimization against $\nu$ ) as well as dividing surface (by minimization against $\hat{F}$ ). The unique result of the minimization against $\nu$ is the MST correlation function, which is then seen as the only natural choice for the design of optimal numerical algorithms. Henceforth, we restrict our attention to this particular correlation function. 


\section{A CASE WITH $\sup \bar{C}_{F}^{\mathrm{MST}}(\omega)>\bar{C}_{F}^{\mathrm{MST}}(0)$ : THE SYMMETRIC RECTANGULAR BARRIER}

To demonstrate that recrossing flux cannot in principle be canceled by optimizing the correlation function and the dividing surface, we need to construct a simple example for which

$$
\sup _{\omega \in \mathbb{R}} \bar{C}_{F}^{\mathrm{MST}}(\omega)>\bar{C}_{F}^{\mathrm{MST}}(0) .
$$

Then, according to Eqs. (8) and (10), no choice of correlation function that completely removes the recrossing exists. The first example we treat is the rectangular potential barrier, which is described by the function

$$
V(x)= \begin{cases}V_{0}, & \text { if }|x| \leq a \\ 0, & \text { otherwise }\end{cases}
$$

with $V_{0}>0$ and $a>0$. The parameters for the barrier are $V_{0}=0.425 \mathrm{eV}, a=1.36$ a.u., and $m_{0}=1060$ a.u.. They have been chosen to correspond approximately to the $\mathrm{H}+\mathrm{H}_{2}$ reaction [19]. The optimal dividing surface is located at $x=0$, by symmetry arguments and the fact that the potential is non-increasing to the left and the right of the origin.

In order to determine the flux matrix elements in energy space, we need the properly normalized eigenfunctions for the rectangular barrier. Inside the barrier region, the symmetric and antisymmetric eigenfunctions have the form

$$
\begin{aligned}
& \psi_{+}(x)=A \cos \left(k^{\prime} x\right), \text { for even parity, } \\
& \psi_{-}(x)=B \sin \left(k^{\prime} x\right), \text { for odd parity }
\end{aligned}
$$

with $k^{\prime}$ defined by the equation $\hbar k^{\prime}=\sqrt{2 m_{0}\left(E-V_{0}\right)}$. Outside the barrier region, the eigenfunctions have the form

$$
\begin{aligned}
& \psi_{+}(x)=A_{1} \cos (k x)+A_{2} \sin (k|x|), \\
& \psi_{-}(x)=B_{1} \operatorname{sign}(x) \cos (k x)+B_{2} \sin (k x),
\end{aligned}
$$

with $k$ defined by the equation $\hbar k=\sqrt{2 m_{0} E}$. The determination of the coefficients appearing in the previous paragraph is a well-known matter [12]. One utilizes the conditions of continuity of the eigenfunctions and their derivatives at the points $x= \pm a$ as well as the normalization condition in energy space, which reads

$$
\left|A_{1}\right|^{2}+\left|A_{2}\right|^{2}=\left|B_{1}\right|^{2}+\left|B_{2}\right|^{2}=\frac{1}{\pi}\left(\frac{m_{0}}{2 \hbar^{2} E}\right)^{1 / 2} .
$$


Laborious yet straightforward calculations lead to the following expressions for the coefficients $A$ and $B$ (now, regarded as functions of the energy $E$ ):

$$
\left\{\begin{array}{l}
A(E)=\sqrt{\frac{m_{0} k}{\pi \hbar^{2}}}\left[k^{\prime 2} \sin \left(k^{\prime} a\right)^{2}+k^{2} \cos \left(k^{\prime} a\right)^{2}\right]^{-1 / 2}, \\
B(E)=\sqrt{\frac{m_{0} k}{\pi \hbar^{2}}}\left[k^{\prime 2} \cos \left(k^{\prime} a\right)^{2}+k^{2} \sin \left(k^{\prime} a\right)^{2}\right]^{-1 / 2} .
\end{array}\right.
$$

In order to evaluate the square amplitude of the flux matrix elements, because each energy level is doubly degenerate, we must sum up the contributions of all pairs, so that we have

$$
\begin{gathered}
\left|\left\langle E|\hat{F}| E^{\prime}\right\rangle\right|^{2}=\left|\left\langle E,+|\hat{F}| E^{\prime},+\right\rangle\right|^{2}+\left|\left\langle E,+|\hat{F}| E^{\prime},-\right\rangle\right|^{2} \\
+\left|\left\langle E,-|\hat{F}| E^{\prime},+\right\rangle\right|^{2}+\left|\left\langle E,-|\hat{F}| E^{\prime},-\right\rangle\right|^{2}
\end{gathered}
$$

One easily shows that

$$
\begin{aligned}
\left|\left\langle E|\hat{F}| E^{\prime}\right\rangle\right|^{2}= & \frac{1}{2 m_{0}}\left[A(E)^{2} B\left(E^{\prime}\right)^{2}\left(E^{\prime}-V_{0}\right)\right. \\
+ & \left.A\left(E^{\prime}\right)^{2} B(E)^{2}\left(E-V_{0}\right)\right] .
\end{aligned}
$$

It appears useful to recast Eq. (17) in a form that relates to the matrix elements for a free particle. As such, we introduce the functions

$$
\begin{aligned}
& F_{1}(E)=\pi \hbar A(E)^{2} \sqrt{2 E / m_{0}} \\
& F_{2}(E)=\pi \hbar B(E)^{2}\left(E-V_{0}\right) \sqrt{2 /\left(m_{0} E\right)}
\end{aligned}
$$

and write

$$
\begin{aligned}
& \left|\left\langle E|\hat{F}| E^{\prime}\right\rangle\right|^{2}=\frac{1}{(2 \pi \hbar)^{2}} \\
& \times\left[\sqrt{\frac{E^{\prime}}{E}} F_{1}(E) F_{2}\left(E^{\prime}\right)+\sqrt{\frac{E}{E^{\prime}}} F_{2}(E) F_{1}\left(E^{\prime}\right)\right] .
\end{aligned}
$$

In the free particle limit, the functions $F_{1}(E)$ and $F_{2}(E)$ become constant and equal to 1 .

We mention that, due to the special form of the matrix elements $\left|\left\langle E|\hat{F}| E^{\prime}\right\rangle\right|^{2}$, the double integral over the energy space appearing in appearing in Eq. 2 is in reality a sum of products of one-dimensional integrals. Accordingly, introducing the complex functions

$$
R_{1}\left(\beta_{c}\right)=\frac{1}{2 \pi \hbar} \int_{0}^{\infty} e^{-\beta_{c} E} E^{-1 / 2} F_{1}(E) d E
$$

and

$$
R_{2}\left(\beta_{c}\right)=\frac{1}{2 \pi \hbar} \int_{0}^{\infty} e^{-\beta_{c} E} E^{1 / 2} F_{2}(E) d E
$$




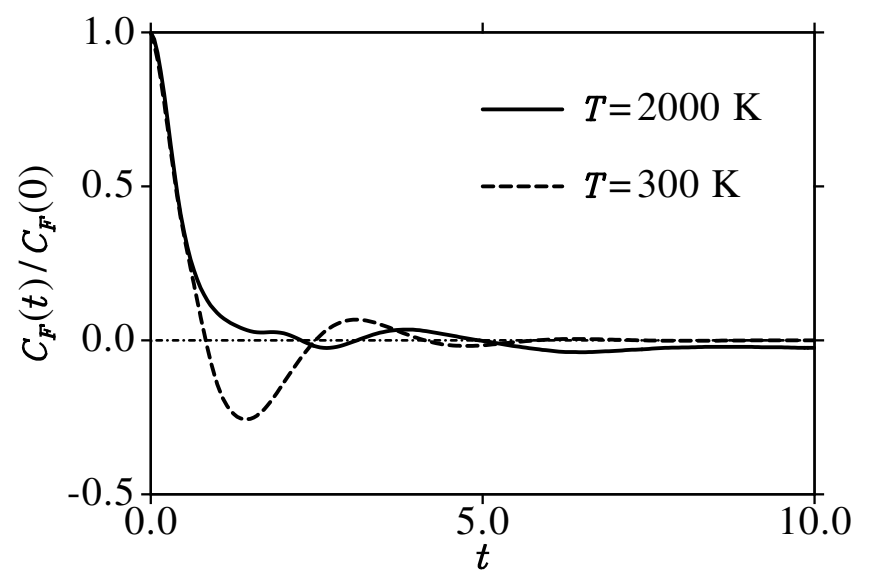

FIG. 1: MST correlation functions for the rectangular barrier at different temperatures. The time is given in units of $\beta \hbar$. Notice the long negative tail at high temperature. The impact of the tails on the computation of the reaction rates can be better understood by looking at the spectral functions, which are plotted in Fig. 2.

we obtain

$$
C_{F}^{\mathrm{MST}}(t)=2 \operatorname{Re}\left[R_{1}(\beta / 2-i t / \hbar) R_{2}(\beta / 2+i t / \hbar)\right]
$$

The MST correlation functions for the rectangular barrier are plotted in Fig. 1. At high temperature, they exhibit a long negative tail that is associated with the low frequency maximum of the corresponding spectral functions plotted in Fig. 2. Provided that the input information is represented by the moments of the spectral function, the reconstruction of the spectral functions is more accurate around the recrossing maxima and less accurate at the origin, according to the relative weights of the respective regions. If the available information comes from real time simulations, the maxima will still cause numerical problems. However, it is the magnitude of the peaks that matters, as opposed to their intensity. As such, a very sharp pick away from the origin will affect more the numerical algorithms based on real-time simulations and less the algorithms based on moment data. Nevertheless, since a direct minimization of the magnitude of the main primary frequencies is not numerically feasible, we have little choice but to minimize their intensity, that is, the quantity

$$
C_{F}^{\mathrm{MST}}(0)=\int_{\mathbb{R}} \bar{C}_{F}^{\mathrm{MST}}(\omega) d \omega
$$

regardless of the type of simulation conducted. 


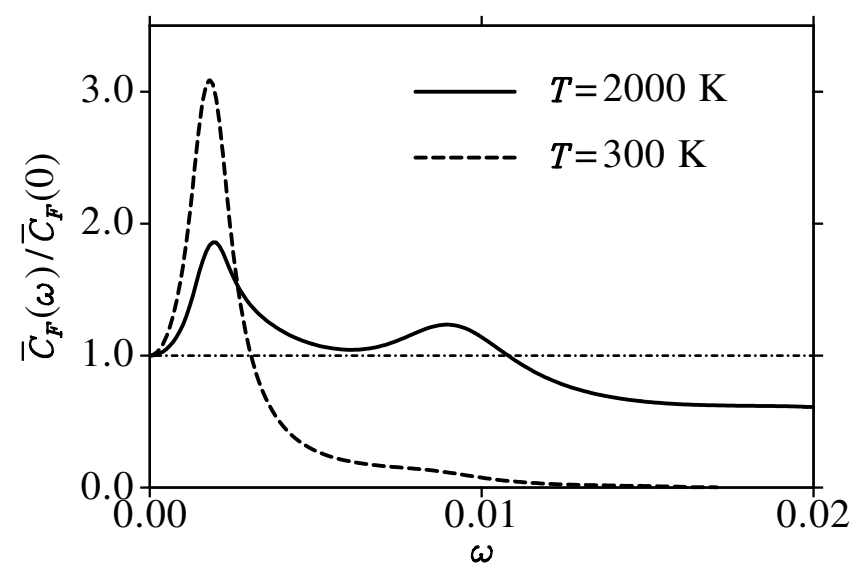

FIG. 2: MST spectral functions for the rectangular barrier at different temperatures. According to Eq. (8), the peaks of the spectral functions away from the origin are responsible for recrossing. We refer to these maxima as the main primary frequencies.

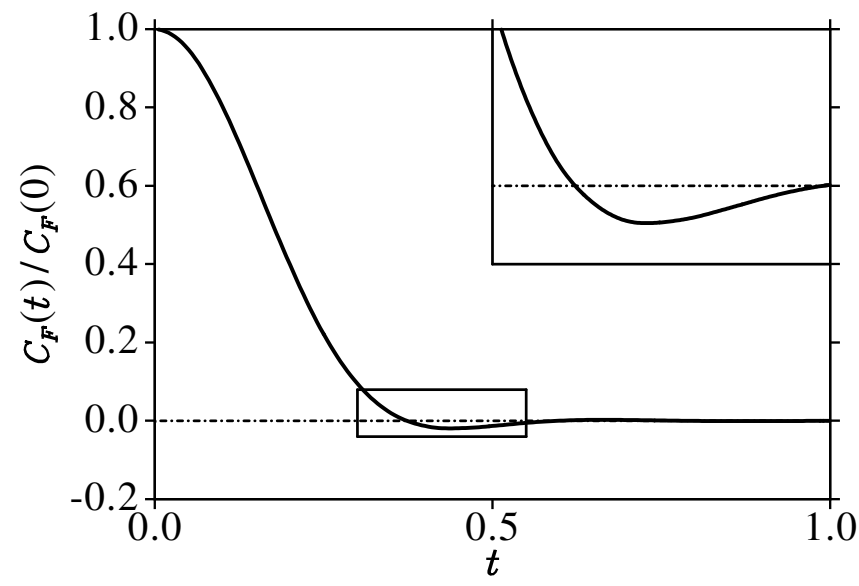

FIG. 3: MST flux-flux correlation function for the one-dimensional symmetric Eckart barrier relative to the value at the origin. The time is given in units of $\hbar \beta$. The temperature is $300 \mathrm{~K}$. The portion of the correlation function that contains the negative part is delimited by a box and magnified in the inset (the proportions have been changed).

IV. A CASE WITH $\sup \bar{C}_{F}^{\mathrm{MST}}(\omega)=\bar{C}_{F}^{\mathrm{MST}}(0)$ : THE SYMMETRIC ECKART BARRIER

If the equality

$$
\sup _{\omega \in \mathbb{R}} \bar{C}_{F}^{\mathrm{MST}}(\omega)=\bar{C}_{F}^{\mathrm{MST}}(0)
$$


holds, that is, if the MST spectral function attains its maximum value at the origin, the optimal correlation function is in general not unique. For numerical algorithms based on imaginary-time data (including moment data), the additional criterion of minimizing the value of the correlation function at the origin uniquely identifies the MST correlation function as the optimal choice [13]. As discussed in the preceding sections, for algorithms based on real-time simulations, a better criterion would be provided by the ratio

$$
p(\nu)=\int_{0}^{\infty}\left|C_{F, \nu}(t)\right| d t / \int_{0}^{\infty} C_{F, \nu}(t) d t,
$$

if it were not for the computational unfeasibility of the criterion. As we shall show for the simple example of the symmetric Eckart barrier, if Eq. (23) is satisfied, then the extent of the residual oscillations, as measured by the ratio $p(\nu)$, constitutes only a small fraction of the actual value of the reaction rate. Thus, the computation of the ratio $p(\nu)$ requires very accurate evaluations of the quantity

$$
\int_{0}^{\infty}\left|C_{F, \nu}(t)\right| d t
$$

so that the error upon the variation of the $d \nu(\lambda)$ be less than the aforementioned small fraction of the reaction rate. It is then obvious that minimizing $p(\nu)$ is pointless: if such an accuracy were achievable, one could just evaluate the reaction rate and not worry about the precise shape of the optimal correlation function.

In order to attain the accuracy necessary to reliably quantify the small recrossing flux, we evaluate the correlation and spectral functions analytically (up to a numerical quadrature). As for the rectangular barrier, the matrix elements $\left|\left\langle E|\hat{F}| E^{\prime}\right\rangle\right|^{2}$ for the flux operator $\hat{F}$ defined by a dividing surface located at the top of the barrier have the form

$$
\begin{aligned}
& \left|\left\langle E|\hat{F}| E^{\prime}\right\rangle\right|^{2}=\frac{1}{(2 \pi \hbar)^{2}} \\
& \times\left[\sqrt{\frac{E^{\prime}}{E}} F_{1}(E) F_{2}\left(E^{\prime}\right)+\sqrt{\frac{E}{E^{\prime}}} F_{2}(E) F_{1}\left(E^{\prime}\right)\right] .
\end{aligned}
$$

The functions $F_{1}(E)$ and $F_{2}(E)$ are defined by

$$
F_{1}(E)=2 \pi^{2} \hbar \sqrt{\frac{2 E}{m_{0}}} \frac{N(\alpha)^{2}}{\left|\Gamma\left(\frac{3}{4}-i \alpha+i \frac{\delta}{2}\right) \Gamma\left(\frac{3}{4}-i \alpha-i \frac{\delta}{2}\right)\right|^{2}}
$$

and

$$
F_{2}(E)=\frac{8 \pi^{2} \hbar^{3} a^{2}}{m_{0} \sqrt{2 m_{0} E}} \frac{N(\alpha)^{2}}{\left|\Gamma\left(\frac{1}{4}-i \alpha+i \frac{\delta}{2}\right) \Gamma\left(\frac{1}{4}-i \alpha-i \frac{\delta}{2}\right)\right|^{2}}
$$




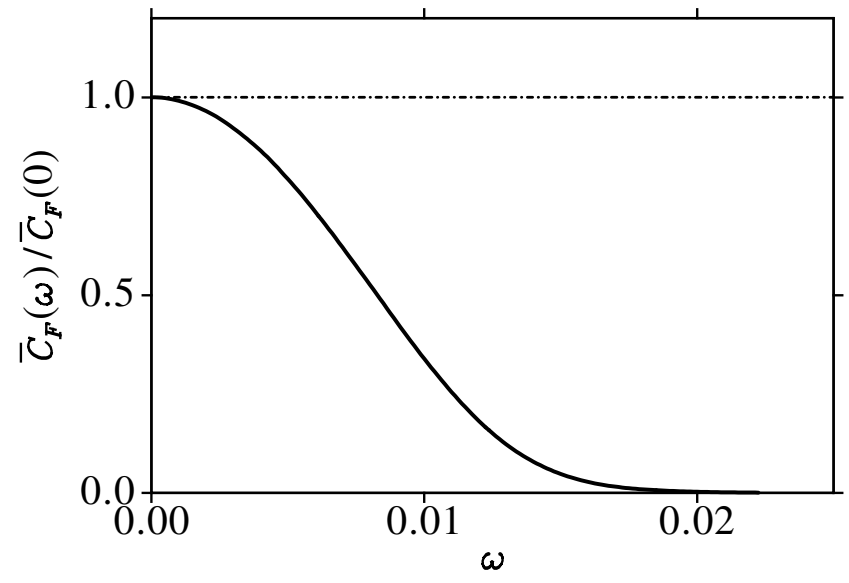

FIG. 4: MST spectral function for the Eckart barrier at the temperature of $T=300 \mathrm{~K}$. Despite the fact that the correlation function decreases monotonically away from the origin, its Fourier transform exhibits slight negative parts, as shown in Fig. 3.

with

$$
\alpha=\frac{1}{2}\left(\frac{2 m_{0} E}{\hbar^{2} a^{2}}\right)^{1 / 2} .
$$

They are derived in the Appendix. In the free particle limit, the functions become $F_{1}(E)=$ $F_{2}(E)=1$.

The MST correlation function at the temperature $T=300 \mathrm{~K}$ is shown in Fig. 3. The parameters for the Eckart barrier are as for the rectangular barrier: $V_{0}=0.425 \mathrm{eV}, a=$ 1.36 a.u., and $m_{0}=1060$ a.u.. Despite the fact that its spectral function has no maximum away from the origin, as shown in Fig. 4, the MST correlation function exhibits a slight negative part that will result in overestimation of the quantum reaction rate for all theories that neglect recrossing. Indeed, apart from numerical errors associated with the specifics of the particular transition state theory, there is a systematic error which, to some extent, can be quantified by the quantity $[p(T)-1] \cdot 100$, where

$$
p(T)=\frac{1}{k(T) Q_{r}(T)} \int_{0}^{\infty}\left|C_{F}^{\mathrm{MST}}(t)\right| d t
$$

The value $[p(T)-1] \cdot 100$ can be regarded as the percent error in the determination of the reaction rate and is plotted in Fig. 5, as a function of temperature.

As argued in the introductory paragraph, further optimization of the correlation function to completely remove the recrossing (if possible at all) is not a computationally optimal strategy. A logical conclusion is that quantum transition state theories must not rely solely 


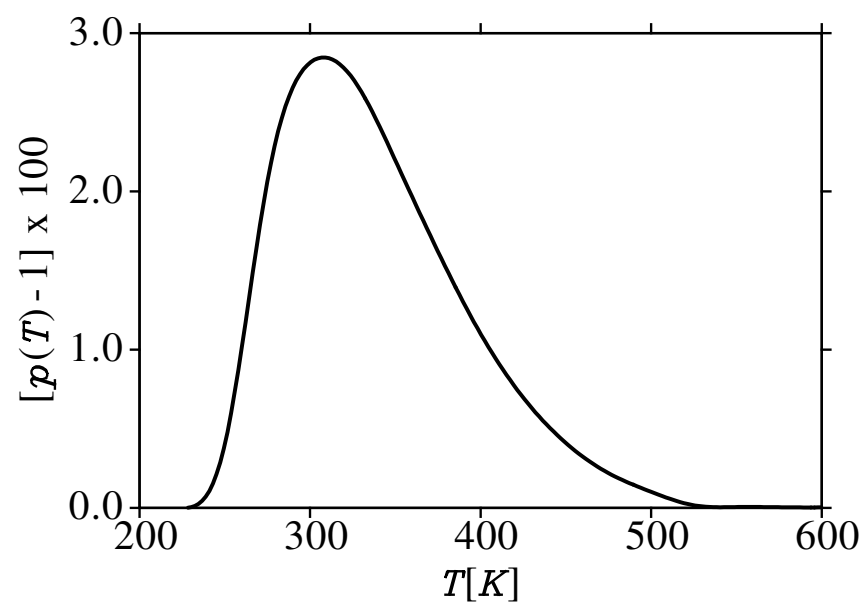

FIG. 5: Overestimation of the quantum reaction rate for the symmetric Eckart barrier as a function of the temperature. The numerical errors of the quantity $[p(T)-1] \cdot 100$ are less than 0.01 , as absolute values. Therefore, the possibility that the negative parts of the TST correlation functions are numerical artifacts must be ruled out.

on the "no recrossing" assumption. Instead, they must provide expressions flexible enough to account at least for small amounts of residual recrossing.

\section{SUMMARY AND CONCLUSIONS}

We have provided an analysis of the issue of recrossing for quantum systems. We have demonstrated that the MST correlation function represents the only reasonable choice with respect to minimizing the extent of recrossing flux. In this respect, we have argued that further optimizing the correlation function to reduce the recrossing flux beyond what the MST correlation function achieves incurs a computational cost that is larger than the cost for the determination of the reaction rate itself. Instead, the residual recrossing flux, which is generally not zero even for the simplest physical systems, is best addressed by designing theories that do not completely rely on the "no recrossing" assumption. A conclusion of our analysis is that it is not possible to obtain a rigorous quantum transition state theory that provides an upper bound to the true reaction rate and that is also computationally useful.

We have also derived the analytic expressions of the flux-flux correlation and spectral functions for the symmetric Eckart and rectangular barriers. Besides their pedagogical value, the analytical formulas are expected to be useful in verifying the stability of Monte 
Carlo algorithms that compute imaginary-time data of use in spectral analysis.

\section{Acknowledgments}

This work was supported in part by the National Science Foundation Grant Number CHE-0345280, the Director, Office of Science, Office of Basic Energy Sciences, Chemical Sciences, Geosciences, and Biosciences Division, U.S. Department of Energy under Contract Number DE AC03-65SF00098, and the U.S.-Israel Binational Science Foundation Award Number 2002170.

\section{APPENDIX A: EVALUATION OF THE PROPAGATOR AND THE FLUX MA- TRIX ELEMENTS IN ENERGY SPACE FOR SYMMETRIC ECKART BARRIER}

Although the initial motivation was to provide mathematical insight into the penetration of a low-energy electron through a barrier [14], the Eckart potential barrier has been more extensively employed in the chemical-physics literature as a benchmark for various quantum transition state theories (TST) [9, 15-19]. The symmetric Eckart barrier potential is given by the expression

$$
V(x)=V_{0} / \cosh (a x)^{2}
$$

and depends on the two positive parameters $V_{0}$ and $a$, which establish the height and the width of the barrier. The following more compact notation is needed:

$$
\delta=\frac{1}{2}\left(\frac{8 m_{0} V_{0}}{\hbar^{2} a^{2}}-1\right)^{1 / 2}, \quad \alpha=\frac{1}{2}\left(\frac{2 m_{0} E}{\hbar^{2} a^{2}}\right)^{1 / 2}
$$

and $\xi=-\exp (2 a x)$. Notice that $\delta$ can be either real or purely imaginary. As shown by Eckart [see Eq. (8) of Ref. 14], one of the eigenfunctions of the Schrödinger equation corresponding to the energy $E \geq 0$ is given in terms of the hypergeometric function $F(a, b ; c ; x)$ by the equation

$$
u_{\alpha}(x)=(-\xi)^{i \alpha} F\left(\frac{1}{2}+i \delta,-\frac{1}{2}-i \delta ; 1-2 i \alpha ; \frac{1}{1-\xi}\right)
$$

For $E>0$, there are two degenerate eigenfunctions, with the second obtained by replacing $\xi$ with $1 / \xi$ in Eq. (A3). This follows from the symmetry of potential $V(x)$ at inversion of 
coordinate $x \mapsto-x$. Therefore, replacing $\xi=-\exp (2 a x)$ with $-\exp (-2 a x)=1 / \xi$ must produce the second eigenfunction.

The asymptotic behavior in the limit $x \rightarrow \infty$ can be established from Eq. (A3) and is given by

$$
u_{\alpha}(x) \sim(-\xi)^{i \alpha}=e^{2 i \alpha a x}
$$

because $F(a, b ; c ; 0)=1$. The behavior in the limit $x \rightarrow-\infty$ cannot be determined at once from Eq. (A3) because the series $F(a, b ; c ; 1)$ diverges. However, by utilizing another form of the solution (which is still given by one of the 24 ways in which the solution of the hypergeometric equation can be expressed), Eckart showed that the asymptotic behavior of Eq. (A3) in the limit $x \rightarrow-\infty$ is given by

$$
u_{\alpha}(x) \sim a_{1} e^{2 i \alpha a x}+a_{2} e^{-2 i \alpha a x}
$$

where

and

$$
a_{1}=\frac{\Gamma(1-2 i \alpha) \Gamma(-2 i \alpha)}{\Gamma\left(\frac{1}{2}-i \delta-2 i \alpha\right) \Gamma\left(\frac{1}{2}+i \delta-2 i \alpha\right)}
$$

$$
a_{2}=\frac{\Gamma(1-2 i \alpha) \Gamma(2 i \alpha)}{\Gamma\left(\frac{1}{2}-i \delta\right) \Gamma\left(\frac{1}{2}+i \delta\right)} .
$$

Notice that $\left|a_{1}\right|^{2}=1+\left|a_{2}\right|^{2}$, a relation that has the physical meaning that the sum of the amplitudes of the transmitted and reflected waves must equal the amplitude of the incident wave. Also, by employing the relation $\Gamma(1+z)=z \Gamma(z)$, we see that

$$
a_{2}=-2 i \alpha \frac{|\Gamma(2 i \alpha)|^{2}}{\left|\Gamma\left(\frac{1}{2}-i \delta\right) \Gamma\left(\frac{1}{2}+i \delta\right)\right|},
$$

regardless of whether $\delta$ is purely imaginary or real. Therefore, $a_{2}$ is always purely imaginary.

The normalization coefficient $N(\alpha)$ of the eigenfunctions $u_{\alpha}(x)$ and $u_{\alpha}(-x)$ in the energy space is obtained from the "normalization in a box" requirement

$$
\begin{array}{r}
\lim _{R \rightarrow \infty} \frac{1}{2 R} \int_{-R}^{R}|N(\alpha)|^{2} u_{\alpha}(x)^{*} u_{\alpha}(x) d x \\
=\frac{1}{2 \pi}\left(\frac{m_{0}}{2 \hbar^{2} E}\right)^{1 / 2}=\frac{m_{0}}{4 \pi \hbar^{2} a \alpha}
\end{array}
$$

a relation that is similar to that of a free particle. In the limit $R \rightarrow \infty$, the main contribution to the integral appearing in the preceding equation comes from the asymptotic regions. Thus, we have the equality

$$
|N(\alpha)|^{2} \frac{1}{2}\left(1+\left|a_{1}\right|^{2}+\left|a_{2}\right|^{2}\right)=|N(\alpha)|^{2}\left|a_{1}\right|^{2}=\frac{m_{0}}{4 \pi \hbar^{2} a \alpha}
$$


from which the normalization coefficient can be determined up to an arbitrary phase factor. We shall delay the computation of the normalization coefficient for later, after we obtain a more compact representation of the wavefunctions. For now, we only point out that the eigenfunctions $u_{\alpha}(x)$ and $u_{\alpha}(-x)$ are orthogonal. Again by asymptotic analysis, we compute

$$
\begin{array}{r}
\lim _{R \rightarrow \infty} \frac{1}{2 R} \int_{-R}^{R}|N(\alpha)|^{2} u_{\alpha}(x)^{*} u_{\alpha}(-x) d x \\
=|N(\alpha)|^{2}\left(a_{2}+a_{2}^{*}\right) / 2=0
\end{array}
$$

where we have utilized the previously established fact that $a_{2}$ is purely imaginary.

The eigenfunctions of the symmetric Eckart barrier can be most conveniently expressed in terms of the associated Legendre functions. The connection is given by Eq. 8.702 of Ref. 20, which reads

$$
P_{\nu}^{\mu}(z)=\frac{1}{\Gamma(1-\mu)}\left(\frac{z+1}{z-1}\right)^{\frac{\mu}{2}} F\left(-\nu, \nu+1 ; 1-\mu ; \frac{1-z}{2}\right) .
$$

Straightforward computations lead to the following form for the normalized solutions of the Schrödinger equation

$$
u_{\alpha}( \pm x)=N(\alpha)(-1)^{i \alpha} \Gamma(1-2 i \alpha) P_{-\frac{1}{2}-i \delta}^{2 i \alpha}[ \pm \tanh (a x)]
$$

It is convenient to redefine the normalization coefficient $N(\alpha)$ to encompass the whole prefactor appearing before the associated Legendre functions. Also, because wavefunctions are defined up to a phase factor, that prefactor may arbitrarily be chosen to be positive. We obtain

$$
\begin{array}{r}
N(\alpha)^{2}=\frac{m_{0}}{4 \pi \hbar^{2} a \alpha}\left|\Gamma\left(\frac{1}{2}-i \delta-2 i \alpha\right) \Gamma\left(\frac{1}{2}+i \delta-2 i \alpha\right)\right|^{2} \\
/|\Gamma(-2 i \alpha)|^{2}
\end{array}
$$

Eckart proves that

$$
\begin{array}{r}
\left|\Gamma\left(\frac{1}{2}-i \delta-2 i \alpha\right) \Gamma\left(\frac{1}{2}+i \delta-2 i \alpha\right)\right|^{2} \\
=2 \pi^{2} /[\cosh (4 \pi \alpha)+\cosh (2 \pi \delta)],
\end{array}
$$

whether $\delta$ is real or purely imaginary. Moreover, point 1. of Eq. 8.332 of Ref. 20 says that

$$
|\Gamma(-2 i \alpha)|^{2}=\frac{\pi}{2 \alpha \sinh (2 \pi \alpha)}
$$


Therefore, the normalization coefficient $N(\alpha)$ is given by the expression

$$
N(\alpha)=\left(\frac{m_{0}}{\hbar^{2} a}\right)^{1 / 2}\left[\frac{\sinh (2 \pi \alpha)}{\cosh (4 \pi \alpha)+\cosh (2 \pi \delta)}\right]^{1 / 2},
$$

whereas the two orthonormal eigenfunctions of energy $E=2 \hbar^{2} a^{2} \alpha^{2} / m_{0}$ are

$$
u_{\alpha}( \pm x)=N(\alpha) P_{-\frac{1}{2}-i \delta}^{2 i \alpha}[ \pm \tanh (a x)]
$$

Again, the normalization of the eigenfunctions is in the energy space. Therefore, the propagator for the symmetric Eckart barrier reads

$$
\begin{aligned}
K\left(x, x^{\prime} ; \beta_{c}\right)= & \int_{0}^{\infty} e^{-\beta_{c} E}\left[u_{\alpha}(x)^{*} u_{\alpha}\left(x^{\prime}\right)\right. \\
& \left.+u_{\alpha}(-x)^{*} u_{\alpha}\left(-x^{\prime}\right)\right] d E
\end{aligned}
$$

where $\alpha=\sqrt{m_{0} E /\left(2 \hbar^{2} a^{2}\right)}$ and $\beta_{c}=\beta+i t / \hbar$.

At this point, we mention that the propagator for the symmetric Eckart barrier can also be obtained by path-integral arguments, as recently shown by Guechi and Hammann [21]. Although the derivation is significantly more intricate when compared to the direct spectral analysis, the path-integral construction is extremely rewarding and constitutes an excellent application of the dimensional extension technique utilized by the authors to transform the problem into a Morse potential problem. We point out however that there is a small inconsistency at the very end of the derivation performed in the aforementioned work. The normalization constant obtained by Guechi and Hammann reads

$$
N(\alpha)=\left(\frac{m_{0}}{2 \hbar^{2} a}\right)^{1 / 2} \frac{\sinh (2 \pi \alpha)^{1 / 2}}{\left|\sin \left[\pi\left(\frac{1}{2}+i \delta+i 2 \alpha\right)\right]\right|} .
$$

As pointed out by Eckart in a similar context, the equality

$$
\cosh (4 \pi \alpha)+\cosh (2 \pi \delta)=2\left|\sin \left[\pi\left(\frac{1}{2}+i \delta+i 2 \alpha\right)\right]\right|^{2}
$$

holds only for purely imaginary $\delta$. The right-hand side expression cannot be continued to real values of $\delta$ because the absolute value is not an analytic function. Therefore, Eqs. (A7) and (A10) are equivalent only for purely imaginary $\delta$, whereas for arbitrary values, Eq. (A7) must be utilized. We mention however that this inconsistency does not in any way invalidate the path-integral arguments of Guechi and Hammann, which remain correct. 
Using the analytical expressions of the eigenfunctions $u_{\alpha}( \pm x)$ together with Eq. (16), perhaps more readily after replacing the functions $u_{\alpha}( \pm x)$ with the orthonormal symmetric and antisymmetric combinations

$$
\phi_{\alpha}( \pm x)=\left[u_{\alpha}(x) \pm u_{\alpha}(-x)\right] / \sqrt{2}
$$

we obtain

$$
\begin{aligned}
& \left|\left\langle E|\hat{F}| E^{\prime}\right\rangle\right|^{2}=N(\alpha)^{2} N\left(\alpha^{\prime}\right)^{2} \frac{\hbar^{2} a^{2}}{m_{0}^{2}} \\
& \times\left[\left|P_{-\frac{1}{2}-i \delta}^{2 i \alpha}(0)\right|^{2}\left|\frac{d}{d x} P_{-\frac{1}{2}-i \delta}^{2 i \alpha^{\prime}}(0)\right|^{2}\right. \\
& \left.+\left|P_{-\frac{1}{2}-i \delta}^{2 i \alpha^{\prime}}(0)\right|^{2}\left|\frac{d}{d x} P_{-\frac{1}{2}-i \delta}^{2 i \alpha}(0)\right|^{2}\right] .
\end{aligned}
$$

Of course, the value $\alpha^{\prime}$ is the one corresponding to the energy $E^{\prime}$, as shown by the equation defining $\alpha$, which appears in Eq. (A2).

The special values of the associated Legendre functions and their derivatives at the origin are given in Eq. 8.756 of Ref. 20. The formulas read

$$
P_{\nu}^{\mu}(0)=\frac{2^{\mu} \sqrt{\pi}}{\Gamma\left(\frac{\nu-\mu}{2}+1\right) \Gamma\left(\frac{-\nu-\mu+1}{2}\right)}
$$

and

$$
\frac{d P_{\nu}^{\mu}(0)}{d x}=\frac{2^{\mu+1} \sin \left[\frac{\pi}{2}(\nu+\mu)\right] \Gamma\left(\frac{\nu+\mu}{2}+1\right)}{\sqrt{\pi} \Gamma\left(\frac{\nu-\mu+1}{2}\right)},
$$

respectively. The latter equation can be transformed into a relation resembling the former with the help of the identity $\Gamma(z) \Gamma(1-z)=\pi / \sin (\pi z)$ [see Eq. 8.334, point 3. of Ref. 20]. We obtain

$$
\frac{d P_{\nu}^{\mu}(0)}{d x}=\frac{2^{\mu+1} \sqrt{\pi}}{\Gamma\left(\frac{\nu-\mu+1}{2}\right) \Gamma\left(\frac{-\nu-\mu}{2}\right)} .
$$

The matrix element $\left|\left\langle E|\hat{F}| E^{\prime}\right\rangle\right|^{2}$ takes a particularly simple form if we introduce the positive functions

$$
F_{1}(E)=\frac{2 \pi \hbar a}{m_{0}} \frac{N(\alpha)^{2}}{\left|\Gamma\left(\frac{3}{4}-i \alpha+i \frac{\delta}{2}\right) \Gamma\left(\frac{3}{4}-i \alpha-i \frac{\delta}{2}\right)\right|^{2}}
$$

and

$$
F_{2}(E)=\frac{2 \pi \hbar a}{m_{0}} \frac{N(\alpha)^{2}}{\left|\Gamma\left(\frac{1}{4}-i \alpha+i \frac{\delta}{2}\right) \Gamma\left(\frac{1}{4}-i \alpha-i \frac{\delta}{2}\right)\right|^{2}} .
$$


We thus have

$$
\left|\left\langle E|\hat{F}| E^{\prime}\right\rangle\right|^{2}=F_{1}(E) F_{2}\left(E^{\prime}\right)+F_{2}(E) F_{1}\left(E^{\prime}\right)
$$

As for the rectangular barrier, we recast Eq. (A14) in a form that relates to the matrix elements for a free particle. We redefine the functions $F_{1}(E)$ and $F_{2}(E)$ to read

$$
F_{1}(E)=2 \pi^{2} \hbar \sqrt{\frac{2 E}{m_{0}}} \frac{N(\alpha)^{2}}{\left|\Gamma\left(\frac{3}{4}-i \alpha+i \frac{\delta}{2}\right) \Gamma\left(\frac{3}{4}-i \alpha-i \frac{\delta}{2}\right)\right|^{2}}
$$

and

$$
F_{2}(E)=\frac{8 \pi^{2} \hbar^{3} a^{2}}{m_{0} \sqrt{2 m_{0} E}} \frac{N(\alpha)^{2}}{\left|\Gamma\left(\frac{1}{4}-i \alpha+i \frac{\delta}{2}\right) \Gamma\left(\frac{1}{4}-i \alpha-i \frac{\delta}{2}\right)\right|^{2}} .
$$

Straightforward calculations lead to

$$
\begin{aligned}
& \left|\left\langle E|\hat{F}| E^{\prime}\right\rangle\right|^{2}=\frac{1}{(2 \pi \hbar)^{2}} \\
& \times\left[\sqrt{\frac{E^{\prime}}{E}} F_{1}(E) F_{2}\left(E^{\prime}\right)+\sqrt{\frac{E}{E^{\prime}}} F_{2}(E) F_{1}\left(E^{\prime}\right)\right] .
\end{aligned}
$$

The free particle limit of Eq. (A17) can be readily obtained because $F_{1}(E)=F_{2}(E)=1$ in this limit. 
[1] E. P. Wigner, J. Chem. Phys. 5 (1937) 720.

[2] E. P. Wigner, Trans. Faraday Soc. 29 (1938) 29.

[3] J. C. Keck, Adv. Chem. Phys. 13 (1967) 85.

[4] P. Pechukas, F. J. McLafferty, J. Chem. Phys. 58 (1973) 1622.

[5] W. H. Miller, J. Phys. Chem. A 102 (1998) 793.

[6] B. C. Garrett, Theor. Chem. Acc. 103 (2000) 200.

[7] P. Pechukas, F. J. McLafferty, Chem. Phys. Lett. 27 (1973) 511.

[8] W. H. Miller, J. Chem. Phys. 61 (1974) 1823.

[9] W. H. Miller, S. D. Schwartz, J. W. Tromp, J. Chem. Phys. 79 (1983) 4889.

[10] T. Yamamoto, J. Chem. Phys. 33 (1960) 281.

[11] C. Predescu, Phys. Rev. E 70 (2004) 066705.

[12] E. Merzbacher, Quantum Mechanics, John-Wiley \& Sons, New York, 1998.

[13] C. Predescu, W. H. Miller, J. Phys. Chem. B 109 (2005) 6491.

[14] C. Eckart, Phys. Rev. 35 (1930) 1303.

[15] G. A. Voth, D. Chandler, W. H. Miller, J. Chem. Phys. 91 (1989) 7749.

[16] N. F. Hansen, H. C. Andersen, J. Chem. Phys. 101 (1994) 6032.

[17] G. K. Schenter, G. Mills, H. Jónsson, J. Chem. Phys. 101 (1994) 8964.

[18] E. Pollak, J. L. Liao, J. Chem. Phys. 108 (1998) 2733.

[19] W. H. Miller, Y. Zhao, M. Ceotto, S. Yang, J. Chem. Phys. 119 (2003) 1329.

[20] I. S. Gradshteyn, I. M. Ryzhik, Table of Integrals, Series, and Products, Fifth Edition, ed. Alan Jeffrey, Academic Press, London, 1994.

[21] L. Guechi, T. F. Hammann, Il Nuovo Cimento B 115 (2000) 123. 


\section{FIGURE CAPTIONS}

Fig 1. MST correlation functions for the rectangular barrier at different temperatures. The time is given in units of $\beta \hbar$. Notice the long negative tail at high temperature. The impact of the tails on the computation of the reaction rates can be better understood by looking at the spectral functions, which are plotted in Fig. 2.

Fig 2. MST spectral functions for the rectangular barrier at different temperatures. According to Eq. (8), the peaks of the spectral functions away from the origin are responsible for recrossing. We refer to these maxima as the main primary frequencies.

Fig 3. MST flux-flux correlation function for the one-dimensional symmetric Eckart barrier relative to the value at the origin. The time is given in units of $\hbar \beta$. The temperature is $300 \mathrm{~K}$. The portion of the correlation function that contains the negative part is delimited by a box and magnified in the inset (the proportions have been changed).

Fig 4. MST spectral function for the Eckart barrier at the temperature of $T=300 \mathrm{~K}$. Despite the fact that the correlation function decreases monotonically away from the origin, its Fourier transform exhibits slight negative parts, as shown in Fig. 3.

Fig 5. Overestimation of the quantum reaction rate for the symmetric Eckart barrier as a function of the temperature. The numerical errors of the quantity $[p(T)-1] \cdot 100$ are less than 0.01, as absolute values. Therefore, the possibility that the negative parts of the TST correlation functions are numerical artifacts must be ruled out. 


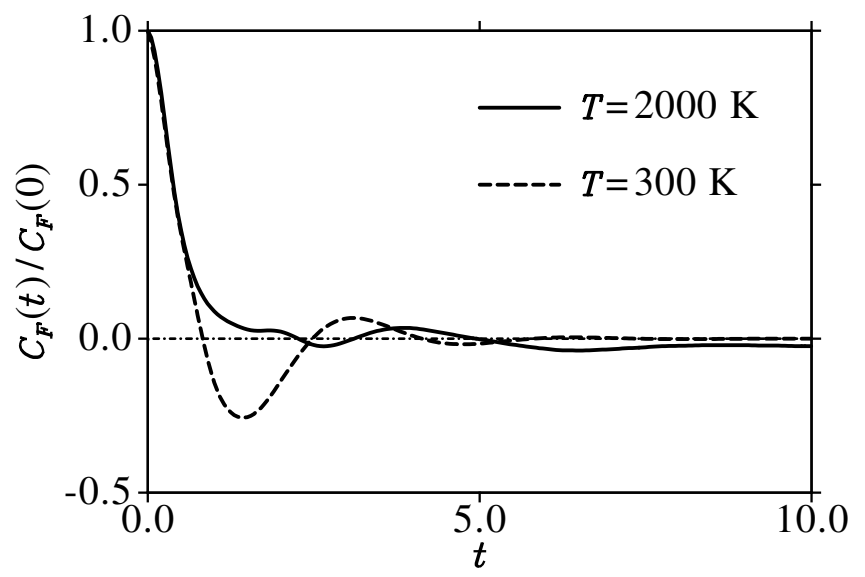

[1] Fig 1 Michael S. Small 


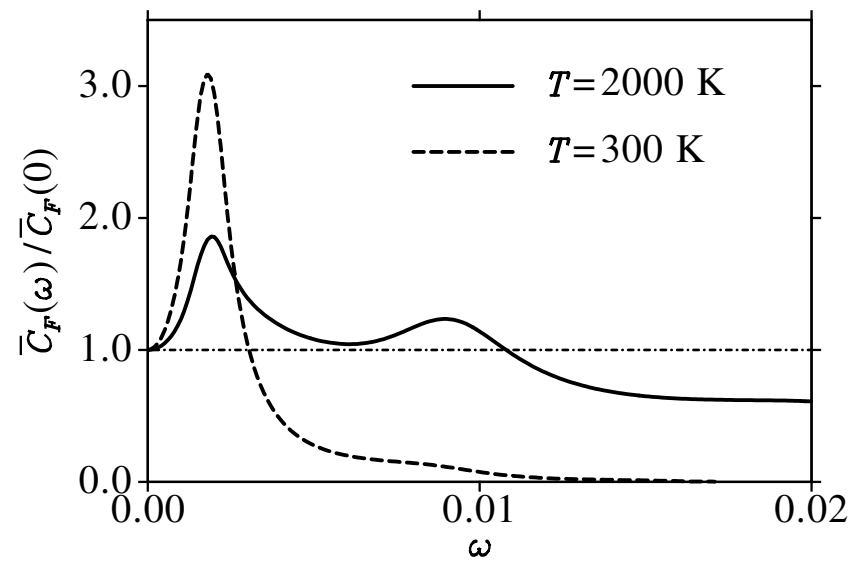

[2] Fig 2 Michael S. Small 


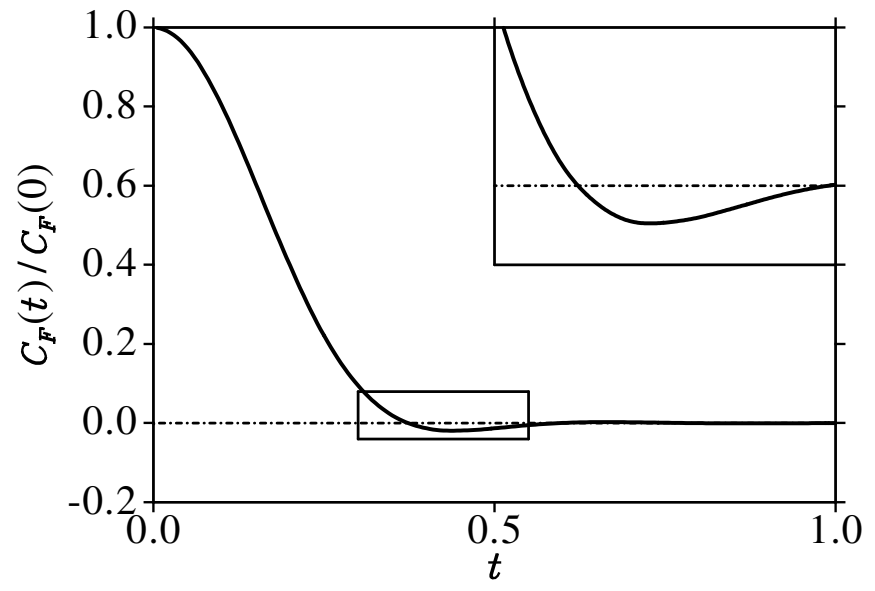

[3] Fig 3 Michael S. Small 


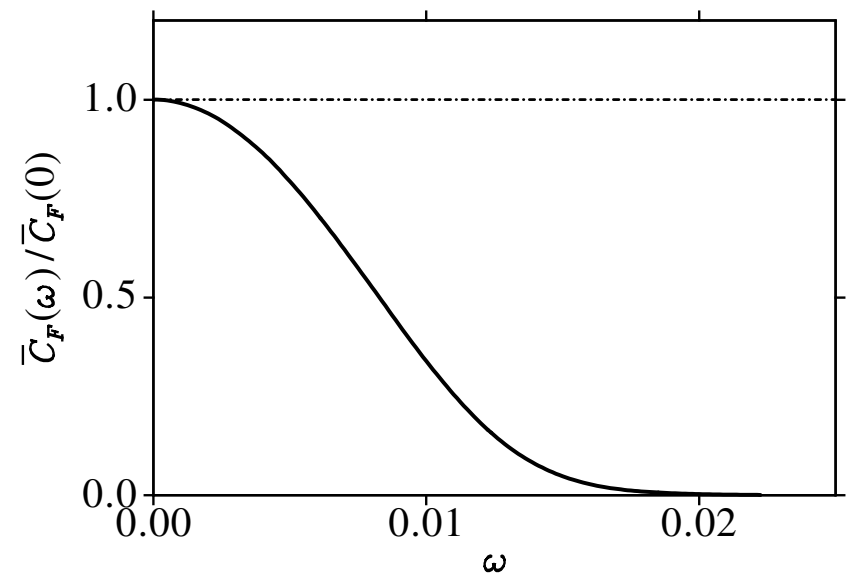

[4] Fig 4 Michael S. Small 


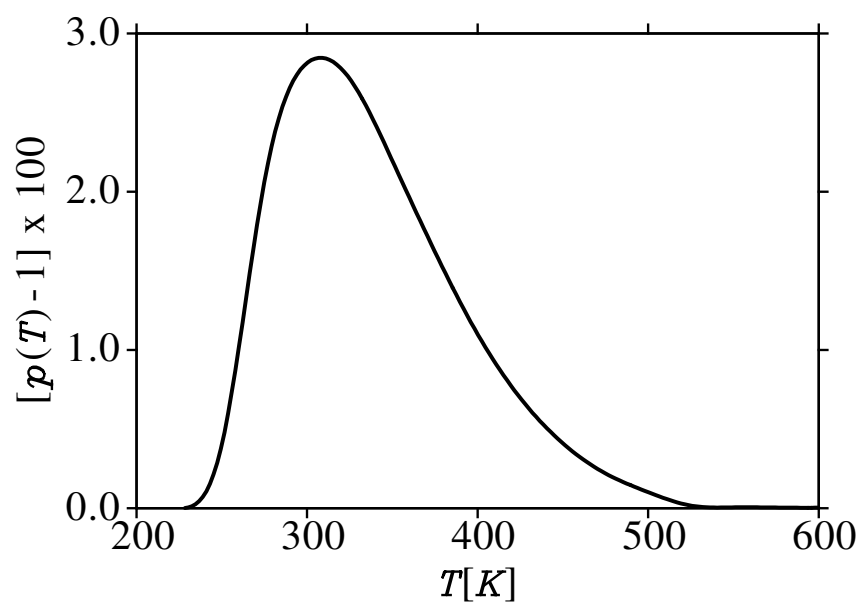

[5] Fig 5 Michael S. Small 